\title{
Inflaton as a pseudo-Goldstone boson of vacuum energy shift symmetry
}

\author{
Ja.V.Balitsky ${ }^{a}$ and V.V.Kiselev ${ }^{a, b, 1}$ \\ ${ }^{a}$ Moscow Institute of Physics and Technology (State University), Russia, 141701, Moscow \\ Region, Dolgoprudny, Institutsky 9 \\ ${ }^{b}$ Russian State Research Center Institute for High Energy Physics (National Research Centre \\ Kurchatov Institute), Russia, 142281, Moscow Region, Protvino, Nauki 1 \\ E-mail: Valery.Kiselev@ihep.ru
}

\begin{abstract}
The exact symmetry induced by the global shift of energy scale for the cosmological constant in the action of matter fields is spontaneously broken by setting the density of real vacuum energy and explicitly broken by the gravity that means the existence of NambuGoldstone boson, which transforms into the pseudo-Goldstone boson due to the gravity. We identify this boson with the inflaton in the Einstein frame of action for the fields, while the breaking is induced by a non-minimal interaction of boson with the scalar curvature in the Jordan frame. The role of Galilean symmetry of field equation is emphasized in the procedure of fixing some terms in the bare action.
\end{abstract}

\footnotetext{
${ }^{1}$ Corresponding author.
} 


\section{Contents}

1 Introduction and the exact global symmetry 1

2 Couplings and symmetry breaking $\quad 3$

3 Matching to the inflaton $\quad 5$

3.1 The effective potential induced by gravitational loops 5

$\begin{array}{lll}3.2 & \text { Transformations of fields } & 7\end{array}$

\section{Introduction and the exact global symmetry}

If the gravity is switched off, then the physics is indifferent with respect to an absolute value of vacuum energy density $\rho_{\Lambda}=\Lambda^{4}$, and the global shift of scale $\Lambda \mapsto \Lambda+\Lambda_{c}$ conserves nongravitational forces, since it corresponds to the choice of minimal-energy prescription or its renormalization, which is irrelevant to the dynamics as constrained by interactions excepting the gravity. Therefore, the non-gravitational action incorporates the global shift symmetry of cosmological constant associated with the vacuum energy-density ${ }^{1}$,

$$
\Lambda \mapsto \Lambda+\Lambda_{c} .
$$

However, in the real world the density of vacuum energy is not arbitrary, it is definite, hence, the global symmetry of non-gravitational forces is spontaneously broken, since the laws of nongravitational dynamics are not changed under this fixing of cosmological constant ${ }^{2}$. Then, the Goldstone theorem $[4,5]$ states that the global parameter of continuous symmetry transforms into the local massless field $\phi(x)$, the Nambu-Goldstone boson, interacting with other fields and conserving the symmetry of action under the global shift symmetry

$$
\phi(x) \mapsto \phi(x)+f_{G} u,
$$

where $u$ is the dimensionless parameter of global symmetry, while $f_{G}$ is the dimensional constant of energy. The value of $f_{G}$ is fixed by setting the canonical expression for the Lagrangian of free field $\phi: \mathcal{L}_{0}=\frac{1}{2}\left(\partial_{\mu} \phi\right)^{2}$. The interaction of such Goldstone boson with the matter can include derivative terms, for instance ${ }^{3}$,

$$
\mathcal{L}=\frac{1}{2}\left(\partial_{\mu} \phi\right)^{2}+b_{e} \partial_{\mu} \phi j^{\mu}+\frac{1}{2} b_{T} \partial_{\mu} \phi \partial_{\nu} \phi T^{\mu \nu}+\ldots
$$

where $j^{\mu}$ is a vector current, which should be bilinear in the matter fields at least, $T^{\mu \nu}$ is a symmetric tensor, which should be linear in the matter fields at least, and so on.

\footnotetext{
${ }^{1}$ See treatments of the cosmological constant problem in [1, 2]. The role of zero-point modes of quantum fields for a determination of vacuum density of energy was first discussed in [3].

${ }^{2}$ We suggest that the super-symmetry (SUSY) is inherently related with the gravity, since the local SUSY gives the super-gravity. See also further discussion in the text.

${ }^{3}$ We exclude higher derivatives to hold the ordinary causality, when the maximal order of differentiation in field equations should not exceed 2.
} 
The global symmetry (1.2) generates the Noether current in the Minkowski space-time

$$
K^{\mu}=\frac{\partial \mathcal{L}}{\partial \partial_{\mu} \phi} \frac{\partial \phi}{\partial u}=f_{G}\left\{\partial^{\mu} \phi+b_{e} j^{\mu}+b_{T} \partial_{\nu} \phi T^{\mu \nu}\right\} .
$$

This current is conserved in the case of absence of gravity, of course. Moreover, the conservation law $\partial_{\mu} K^{\mu}=0$ coincides with the field equation for $\phi$. It is important that in the absence of sources $(j=T=0)$ or at $b_{e}=b_{T}=0$ there is the solution linear in coordinates

$$
\phi=f_{G} k_{\mu} x^{\mu}+\phi_{0}
$$

which conserves the translational invariance of Minkowski space-time, since the global translation $x^{\mu} \mapsto x^{\mu}+a^{\mu}$ generates the global shift $\phi(x) \mapsto \phi(x)+f_{G} k_{\mu} a^{\mu}$, that is the symmetry of action with the Goldstone boson. Solution (1.5) remains valid for the case of non-zero sources, if only the sources are the conserved tensors ${ }^{4}$

$$
\nabla_{\mu} j^{\mu}=0, \quad \nabla_{\mu} T^{\mu \nu}=0 .
$$

Moreover, expression (1.5) corresponds to the classical background for the field of massless quanta for $\phi$. In addition, the expansion over symmetric tensors in (1.3) should be truncated by the shown tensor of second rank, since the absolute conservation laws of real word point to that $j^{\mu}$ is the electric current, while $T^{\mu \nu}$ is the energy-momentum tensor ${ }^{5}$, and we have not get any other conserved symmetric tensors. Then, a non-zero global charge appears at $k^{2}>0$, when in the system $k_{\mu}=\left(k_{0}, \mathbf{0}\right)$ it can be easily calculated, while the boson action with (1.5) is reduced to the following:

$$
S_{\phi}=\left.\int d^{4} x \mathcal{L}\right|_{(1.5)}=\int d t\left\{\frac{1}{2} f_{G}^{2} k^{2}\left(V+b_{T} E\right)+b_{e} f_{G} \sqrt{k^{2}} Q\right\},
$$

where $E$ is the energy of sources in the whole volume $V$ and $Q$ is their electric charge in the same volume. For the vacuum $Q=0, E=\rho_{\Lambda} V$ and we get the global shift for the density of vacuum energy: $\rho_{\Lambda} \mapsto \rho_{\Lambda}-\frac{1}{2} f_{G}^{2} k^{2}\left(1+b_{T} \rho_{\Lambda}\right)$.

The sense of solution (1.5) at $k^{2}>0$ is clear in the inertial system wherein $k^{\mu}=\left(k_{0}, \mathbf{0}\right)$ : at the trivial potential the field of Goldstone boson can move in space homogeneously and isotropically at a constant velocity even when the boson interacts with the conserved sources. Cases of $k^{2}=0$ and $k^{2}<0$ break the mentioned homogeneity and isotropy of space.

For the free Goldstone boson, its energy-momentum tensor under (1.5) trivially takes the ordinary form

$$
t_{\mu_{\nu}}^{(0)}=\left(k_{\mu} k_{\nu}-\frac{1}{2} k^{2} g_{\mu \nu}\right) f_{G}^{2},
$$

that yields the pressure equal to the energy density $p_{0}=\rho_{0}=\frac{1}{2} k^{2} f_{G}^{2}$ in the system $k^{\mu}=$ $\left(k_{0}, \mathbf{0}\right)$. Such the equation of state gives the "stiff matter". The interactions significantly change the value of parameter $w=p / \rho$, of course.

Let us emphasize that solution (1.5) can be interpreted as the introduction of symmetry for the field equations in the form

$$
\phi(x) \mapsto \phi(x)+f_{G} k_{\mu} x^{\mu}+\phi_{0},
$$

\footnotetext{
${ }^{4}$ For the sake of straightforward extension to the case of curved space-time, we use the general covariant derivative $\nabla_{\mu}$ instead of partial derivative of Descartes coordinates in Minkowskian space-time.

${ }^{5}$ The energy-momentum tensor should include the contribution of field $\phi$ itself, that can produce a recurrence.
} 
which has been called the "galilean" invariance [6, 7]. The corresponding scalar field possessing the symmetry of (1.7) of its field equations is referred as the Galileon and it is considered in the gravity modification involving the scalar degree of freedom, so that it can incorporate some higher derivative terms of the field in its action, but the field equations remain the second order equations due to the specified symmetric form of action. In this way, the symmetry permits three higher derivative terms in the action with fixed structures [6]. In addition, by construction the Galileon postulates the decoupling limit: it interacts with the matter only, in the form of contact term of the field with the trace of energy-momentum tensor. We will see that both features of Galileon, i.e. the higher derivative action terms and decoupling from the gravity, are beyond the scope of current study. Thus, we treat the Galilean symmetry of field equation in the very different aspect, whereas the global shift symmetry is broken due to the coupling to the gravity, while the higher derivative terms of self-action are not involved. A study of comparison and connection of our model with the Galileon, especially with the cosmology due to the Galileon [8], will be given elsewhere. However, we emphasize that the Galilean symmetry makes our further constructions to be uniquely definite, since it restricts opportunities in choice of interaction terms.

\section{Couplings and symmetry breaking}

Coupling constants $b_{e, T}$ determine the strengthes of interactions. Note that global field possesses zero interactions. Moreover, the term with $b_{e}$ does not influences the equations of motion. Nevertheless, due to the dimensional analysis it can be represented as the quantity proportional to an inverse scale of energy,

$$
b_{e}=\frac{\kappa}{M}, \quad \kappa= \pm 1,0,
$$

so that $M$ sets the scale of dimensional expansion for the interaction operators, hence,

$$
b_{T} \sim \frac{1}{M^{4}} .
$$

The gravity does not influence the conservation laws (1.6), but it breaks down the global symmetry (1.2). The minimal way of gravitational interaction with the matter conserves the global symmetry of boson vertices. Therefore, we expect that the gravity has to involve non-minimal terms in order to break down the global symmetry.

Suggest that the symmetry is restored in the limit of zero scalar curvature, $\mathcal{R} \rightarrow 0$. In the simplest case the interaction of $\phi$ with the gravity is induced by the Lagrangian linear in both the curvature and field, that explicitly breaks the global symmetry ${ }^{6}$,

$$
\mathcal{L}^{\text {int }}=-\frac{1}{2} b_{\xi} \phi \mathcal{R}
$$

because the shift introduces the finite renormalization of gravitation action itself ${ }^{7}$ :

$$
\mathcal{L}_{G}=-\frac{1}{2} \mathcal{R} \mapsto-\frac{1}{2} \mathcal{R}\left(1+b_{\xi} f_{G} u\right)
$$

\footnotetext{
${ }^{6}$ The linear contact interaction of boson with the scalar curvature conserves the "galilean" symmetry (1.7) of boson field equation. Thus, the galilean symmetry uniquely fixes the linear interaction.

${ }^{7}$ In units $8 \pi G=1$, where $G$ is the Newton constant.
} 
Such the breaking could be called "natural", since the only effect it causes is the change of gravity strength.

Switching on the gravity involves a new symmetric interaction in the form

$$
\mathcal{L}_{S G}=\frac{1}{2} b_{G} \partial_{\mu} \phi \partial_{\nu} \phi\left(\mathcal{R}^{\mu \nu}-\frac{1}{2} g^{\mu \nu} \mathcal{R}\right),
$$

due to Bianchi identity $\nabla_{\mu}\left(\mathcal{R}^{\mu \nu}-\frac{1}{2} g^{\mu \nu} \mathcal{R}\right) \equiv 0$. The coupling constant $b_{G}$ scales as

$$
b_{G} \sim \frac{1}{M^{2}} .
$$

Taking into account Einstein equations

$$
\mathcal{R}^{\mu \nu}-\frac{1}{2} g^{\mu \nu} \mathcal{R}=T^{\mu \nu},
$$

we expect that the terms of interaction with the energy-momentum tensor and Ricci tensor should be of the same order, that means $b_{T} \sim b_{G}$ and $M^{2} \sim 1$ in Planckian units. Thus, the scale $M$ in the effective expansion over the derivative operators is of the order of Planck mass.

The impact of operator $\mathcal{L}_{S G}$ on the inflation has been studied in [9], wherein the effect of enhanced gravitational friction during the slow rolling of inflaton field to a potential minimum was pointed out in the case of minimal coupling of the field to the scalar curvature. So, the coupling to the Einstein tensor $R_{\mu \nu}-\frac{1}{2} g_{\mu \nu} R$ preserves the inflation in the range of perturbative regime safely beyond the limit of strong energy scales. Further generalizations of this issue were considered in [10-12]. After the general introduction of $\mathcal{L}_{S G}$ we disregard this term in our study for the sake of simplicity of demonstration of our basic findings in order to focus on a field potential induced by gravitational loops that break the Galilean symmetry of field equations.

Further, as we will show in the next section, gravitational loop-corrections generate the effective potential starting at the quadratic term ${ }^{8}$ in the mass $m \sim b_{\xi}$,

$$
V=\frac{1}{2} m^{2} \phi^{2}+\ldots,
$$

so that in the leading order of consideration the potential is quadratic in the coupling $b_{\xi}$. In the leading approximation of potential (2.2) and in the case of no sources $(j=T=0)$ or at $b_{e}=b_{T}=b_{G}=0$ the field equation reads off

$$
\square \phi=-m^{2} \phi-\frac{1}{2} b_{\xi} \mathcal{R},
$$

resulting in

$$
\nabla_{\mu} K^{\mu}=-f_{G} m^{2} \phi-\frac{1}{2} b_{\xi} f_{G} \mathcal{R}
$$

that represents the ordinary expression for the pseudo-Goldstone boson and it satisfies the limit of exact global symmetry $\partial_{\mu} K^{\mu} \rightarrow 0$ at $b_{\xi} \rightarrow 0$, since $m^{2} \sim b_{\xi}^{2} \rightarrow 0$.

\footnotetext{
${ }^{8}$ In general, a contribution linear in the field gives a global shift associated with a non-zero value of vacuum expectation value for the field $\phi$.
} 


\section{Matching to the inflaton}

\subsection{The effective potential induced by gravitational loops}

The contact term of non-minimal interaction generates the graviton-graviton-scalar vertex $V^{\mu \nu, \alpha \beta}$ in the Minkowskian background (see figure 1 (left)),

$$
\begin{aligned}
& V^{\mu \nu, \alpha \beta}[p+q, p]=\frac{\xi}{2 m_{\mathrm{Pl}}}\left[\left(p^{2}+q \cdot p+q^{2}\right) \eta^{\mu \alpha} \eta^{\nu \beta}+\right. \\
& \left\{(p+q)^{\alpha} p^{\nu} \eta^{\mu \beta}+(p+q)^{\beta} p^{\mu} \eta^{\nu \alpha}-p^{\alpha} p^{\beta} \eta^{\mu \nu}-\right. \\
& \left.(p+q)^{\mu}(p+q)^{\nu} \eta^{\alpha \beta}\right\} \text {, }
\end{aligned}
$$

where we define the graviton as the perturbation of flat metrics $\eta_{\mu \nu}=\operatorname{diag}(1,-1,-1,-1)$ and set $b_{\xi}=\xi m_{\mathrm{Pl}}$, so that $g_{\mu \nu}=\eta_{\mu \nu}+h_{\mu \nu} / m_{\mathrm{Pl}}$ in Planckian units in terms of reduced Planck mass $m_{\mathrm{Pl}}: 8 \pi G m_{\mathrm{Pl}}^{2}=1$.
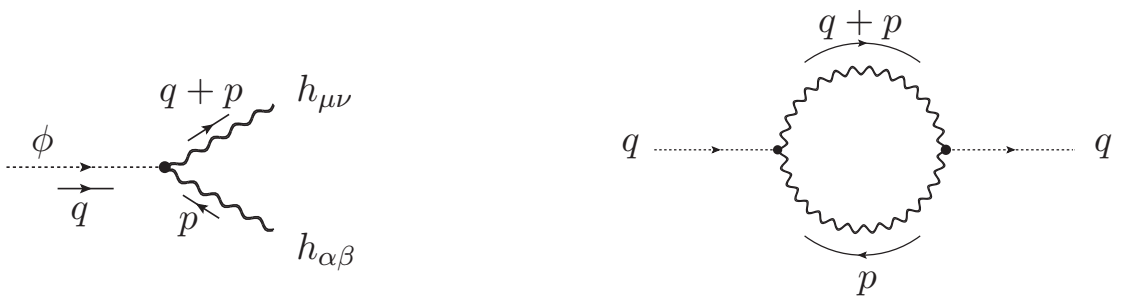

Figure 1. The graviton coupling to the scalar pseudo-Goldstone boson (left) and the graviton loop for the quadratic term of scalar field (right, momenta are labeled).

The graviton propagator is taken in the standard form

$$
\Pi_{\mu \nu, \mu^{\prime} \nu^{\prime}}[p]=\frac{i}{p^{2}+i 0}\left(\eta^{\mu \mu^{\prime}} \eta^{\nu \nu^{\prime}}+\eta^{\mu \nu^{\prime}} \eta^{\nu \mu^{\prime}}-\eta^{\mu \nu} \eta^{\mu^{\prime} \nu^{\prime}}\right)
$$

so that after the Wick rotation to the Euclidean momenta, i.e. at $p^{2}=-p_{E}^{2}, d^{4} p=i d^{4} p_{E}$, $\eta^{\mu \nu} \mapsto-\delta^{\mu \nu}$, we regularize the loop diagram in figure 1 (right)

$$
i \Gamma^{(2)}[q]=\int \frac{d^{4} p}{(2 \pi)^{4}} i V^{\mu \nu, \alpha \beta}[p+q, p] \Pi_{\mu \nu, \mu^{\prime} \nu^{\prime}}[p+q] i V^{\mu^{\prime} \nu^{\prime}, \alpha^{\prime} \beta^{\prime}}[-p-q,-p] \Pi_{\alpha \beta, \alpha^{\prime} \beta^{\prime}}[p]
$$

by introducing the cut-off $\Lambda_{c}$ via $p_{E}^{2}<\Lambda_{c}^{2}$ and after a cumbersome arithmetics we get the quadratic term of effective action

$$
\Gamma[\phi]=\frac{1}{2} \phi^{2} \Gamma^{(2)}[q]
$$

with the effective mass $m$

$$
m^{2}=-\Gamma^{(2)}[0]=c_{2}\left(1-c_{2}^{\prime}\right) \xi^{2} \frac{\Lambda_{c}^{4}}{m_{\mathrm{Pl}}^{2}}, \quad c_{2}=-\frac{39}{64 \pi^{2}},
$$


and the correction to the kinetic term

$$
\frac{\partial^{2} \Gamma^{(2)}[0]}{\partial q^{\mu} \partial q^{\nu}}=\eta_{\mu \nu} k_{2}\left(1-k_{2}^{\prime}\right) \xi^{2} \frac{\Lambda_{c}^{2}}{m_{\mathrm{P} 1}^{2}}, \quad k_{2}=\frac{147}{48 \pi^{2}} .
$$

Here $c_{2}$ and $k_{2}$ are the diagrammatic factors, which were calculated, while $c_{2}^{\prime}$ and $k_{2}^{\prime}$ are some constants of subtraction in the renormalization scheme. At $c_{2}^{\prime}>1$, we get the stable quadratic potential.

Generically, the correction to the kinetic term can be of the order of unit ${ }^{9}$, that means the relation between the coupling constant $b_{\xi}$ and the cut-off scale $\Lambda_{c}$ :

$$
\xi^{2} \sim \frac{m_{\mathrm{Pl}}^{2}}{\Lambda_{c}^{2}}
$$

Therefore, the mass scale of pseudo-Goldstone boson is of the order of loop cut-off,

$$
m \sim \Lambda_{c} .
$$

We suggest that $m \ll m_{\mathrm{Pl}}$, hence, $\xi \gg 1$.

Next, we simplify the consideration by setting the symmetry of effective potential under the inversion $\phi \leftrightarrow-\phi$. It means that all of odd terms in $\phi$ are equal to zero strictly under the renormalization procedure.

Then, we worry about higher powers of $\phi$ in the effective potential. So, we can analogously evaluate term including $\phi^{n}$ by introducing the corresponding cut-off $\Lambda_{n}$ in the Euclidean space. In this way, we can easily evaluate the effective potential as

$$
V_{\mathrm{eff}}=\sum_{n=2 j} \frac{c_{n}}{n !}\left(1-c_{n}^{\prime}\right) \phi^{n} \Lambda_{n}^{4} \frac{\xi^{n}}{m_{\mathrm{Pl}}^{n}}
$$

Here, constants $c_{n}$ are determined by diagrammatic factors, while $c_{n}^{\prime}$ depend on the receipt of renormalization, so that $\left|1-c_{n}^{\prime}\right| \sim 1$. The cut-off scales set the energy factors in diagrams with $n$ gravitons in the loop. Since each contribution determines the independent quantity in the effective potential, the scales of cut-off are generically different for each $n$.

The same diagrams generate contributions, which renormalize the kinetic term,

$$
K_{\mathrm{eff}}=\left(\partial_{\mu} \phi\right)^{2} \sum_{n=2 j} \frac{k_{n}}{n !}\left(1-k_{n}^{\prime}\right) \phi^{n-2} \Lambda_{n}^{2} \frac{\xi^{n}}{m_{\mathrm{Pl}}^{n}},
$$

where $k_{n}$ and $k_{n}^{\prime}$ are diagrammatic and renormalization factors, respectively. We see that the actual expansion of $K_{\text {eff }}$ in $\phi$ corresponds to the expansion in powers of inverse Planck mass $m_{\mathrm{Pl}}$ only if the cut-off scales range as

$$
\Lambda_{n}^{2} \sim m_{\mathrm{Pl}}^{2} \frac{1}{\xi^{n}}
$$

or

$$
\Lambda_{n}^{2} \sim \Lambda_{c}^{2} \frac{1}{\xi^{n-2}}, \quad \text { at } \Lambda_{c}=\Lambda_{2}
$$

\footnotetext{
${ }^{9}$ One could say about the induced kinetic term, but we have already got the propagator of scalar field in the calculation of the 1-loop effective action.
} 
Otherwise the expansion in (3.8) blows up at Planckian values of field. Therefore, the effective potential takes the form

$$
V_{\mathrm{eff}}=\sum_{n=2 j} \frac{c_{n}}{n !}\left(1-\tilde{c}_{n}^{\prime}\right) \frac{\phi^{n}}{m_{\mathrm{Pl}}^{n}} \Lambda_{c}^{2} m_{\mathrm{Pl}}^{2} \frac{1}{\xi^{n-2}},
$$

where $\tilde{c}_{n}^{\prime}$ denote modified renormalization constants in agreement with (3.10).

Thus, setting the true expansion scale to the Planck mass we see that the higher orders of potential at $n>2$ are suppressed as $\xi^{2-n} \ll 1$, at least. We conclude that the leading term of potential is reduced to the quadratic or mass term, indeed.

\subsection{Transformations of fields}

The breaking term corresponds to the scalar field with the non-minimal interaction with the gravity and it sets the action in the Jordan frame of fields in the action. It is well known that the transition to the Einstein frame takes place by the following conformal substitution of metric:

$$
g_{\mu \nu} \mapsto \frac{1}{\Omega(\phi)} g_{\mu \nu}, \quad \Omega(\phi)=1+b_{\xi} \phi,
$$

that results in the action

$$
S=\int d^{4} x \sqrt{-g}\left\{-\frac{1}{2} \mathcal{R}+\frac{1}{2}\left(\partial_{\mu} \phi\right)^{2}\left(\Omega^{-1}+\frac{3}{2}\left(\frac{\partial \ln \Omega}{\partial \phi}\right)^{2}\right)-V_{E}(\phi)\right\},
$$

with the effective potential

$$
V_{E}=\frac{V(\phi)}{\Omega^{2}(\phi)} .
$$

The kinetic term can take the canonical form under the appropriate transformation of scalar field $\phi \mapsto \chi$, that deduces the ordinary gravity with the scalar field. Moreover, the leading quadratic approximation for the potential produces the flat potential $V_{E}$ at $\phi \rightarrow \infty$. Then, we arrive to the inflaton relevant to the real evolution of early Universe [13-16].

Indeed, for the effective potential discussed in the previous subsection

$$
V \mapsto \frac{m^{2}}{2} \phi^{2}, \quad \Omega(\phi) \mapsto 1+\xi \frac{\phi}{m_{\mathrm{Pl}}},
$$

hence,

$$
V_{E}(\phi) \mapsto \frac{m^{2} m_{\mathrm{Pl}}^{2}}{2\left(m_{\mathrm{Pl}}+\xi \phi\right)^{2}} \phi^{2} .
$$

At large couplings $\xi \gg 1$ and positive values of field ${ }^{10}$, the kinetic term becomes canonical under the substitution

$$
\Omega(\phi)=1+\xi \frac{\phi}{m_{\mathrm{Pl}}} \approx \mathrm{e}^{\frac{\chi}{m_{\mathrm{Pl}}} \frac{\sqrt{6}}{3}},
$$

that yields the potential of inflaton $\chi$,

$$
V_{E} \approx \frac{m^{2} m_{\mathrm{Pl}}^{2}}{2 \xi^{2}}\left(1-\mathrm{e}^{-\frac{\chi}{m_{\mathrm{Pl}}} \frac{\sqrt{6}}{3}}\right)^{2} .
$$

\footnotetext{
${ }^{10}$ The general analysis is presented in [17]
} 
Since according to (3.5) and (3.6) $m \sim m_{\mathrm{Pl}} / \xi$, the plateau of potential is given by the mass of pseudo-Goldstone boson,

$$
V_{E}^{\mathrm{inf}}=\Lambda_{\mathrm{inf}}^{4} \sim \frac{m_{\mathrm{Pl}}^{4}}{\xi^{4}},
$$

which is consistent with the observations at $\xi \sim 300$ with the inflation scale $\Lambda_{\text {inf }} \sim 10^{15-16}$ $\mathrm{GeV}$. The inflaton mass is given by

$$
m_{\mathrm{inf}}^{2}=\frac{2 m^{2}}{3 \xi^{2}},
$$

that yields $m_{\mathrm{inf}} \sim 10^{13} \mathrm{GeV}$. Both estimates of $\Lambda_{\mathrm{inf}}$ and $m_{\mathrm{inf}}$ are in agreement with the current phenomenology of Universe inflation.

Thus, at strong coupling $\xi \gg 1$ the model tends to the Starobinsky inflation $[18,19]$ due to the attractor in the space of cosmological parameters [17, 20, 21] relevant to the PLANCK observations [22]. Sub-leading terms of potential could break the attractor conditions, that would be relevant to the recent search for a valuable amplitude of relict gravitational waves, which were induced by the Universe inflation, if the foreground polarization generated by the dust is suppressed in a region of detection. At present, the enforced amplitude of B-mode of cosmic microwave background radiation detected by BICEP2 [23] corresponds to such the secondary foreground produced by the measured dust distribution as the Planck collaboration has reported in [24].

\section{Discussion}

Note that at the inflation stage of Universe expansion the only non-zero component of $\partial_{\mu} \phi$ is a constant temporal component $\partial_{0} \phi \approx$ const., and the interactions of inflaton with the energy-momentum tensor, Ricci tensor and electric current are inessential and they can be neglected, because there are no transformation of energy from the inflaton to the matter, gravity and charges. In contrast, just after the inflation $\partial_{\mu} \phi$ become time-dependent and they constitute sources for the matter production due to the coupling to $T^{\mu \nu}$ (i.e. the reheating of early Universe at $b_{T} \neq 0$ ) and for the direct generation of gravitational waves due to the coupling to the Ricci tensor $R^{\mu \nu}$ (the mechanism of gravitational enhancement at $b_{G} \neq 0$ ). Then, the inhomogeneity of $\phi$ caused by its quantum fluctuations copies itself in the primary inhomogeneity of matter distribution and relict gravitational waves.

Next, the coupling of inflaton to the electric current, $b_{e}$, could be essential for the generation of baryon asymmetry of Universe, if the matter interactions involve appropriate transitions with photons, like, for instance, non-symmetric $p \rightarrow e^{+} \pi^{0} \gamma$ or relevant processes with neutrinos and charged leptons. In this way, Sakharov's conditions [25] are satisfied, since the inflaton evolution is not thermal and it breaks the thermodynamical equilibrium, while the inhomogeneous inflaton becomes the source for processes with the broken both CPinvariance and conservation of baryon number in the matter sector of action. In this respect, a mechanism introducing the scalar gauge field could be actual as considered in [26].

Thus, in the offered mechanism of explicit gravitational breaking of global shift symmetry for the scale of cosmological constant the pseudo-Goldstone nature of inflaton provides us with the reasonable way to describe the actual inflation of early Universe.

Finally, we have to mention that SUSY models usually treat the inflaton as the scalar flat direction of Kähler potential that corresponds to the global shift symmetry, while the superpotential and explicit terms of SUSY breaking generate a non-trivial potential of inflaton 
$[27,28]$. In this way, the inflaton is the pseudo-Goldstone boson, too, but the mechanism is evidently different from the approach considered in this paper.

In addition, the scenario of "natural inflation" [29] ascribes the axion as the inflaton. In this way, the axion is considered as the pseudo-Goldstone boson. Again, we see the treatment, which is very different in its inherent origin.

In fact, both alternative schemes of global symmetry breaking mentioned above are in action even in the case, when the gravity is switched off and the Minkowski space-time is considered, in contrast to our model, which is based on the crucial role of gravity in the symmetry breaking. We think that the global shift symmetry of cosmological constant scale is more relevant to the inflaton dynamics.

The global shift of vacuum energy of matter was also considered in the class of specific theories [30,31], wherein the invariant 4-volume $\sqrt{-g} d^{4} x$ with the metric determinant $g=$ $\operatorname{det} g_{\mu \nu}$ has been modified to the scalar density $\Phi d^{4} x$ with $\Phi$ depending on the first derivatives of scalar fields so that their equations of motion result in the conservation law consistent with the global shift of Lagrangian, that is equivalent to the global shift of cosmological constant. The presence of standard 4-volume terms in the action in addition to $\Phi$ gives the theory with two invariant measures [32]. In this way, one can avoid the cosmological constant problem at all [30]. Further, the involvement of global scale invariance in the double measure theories allows one to construct realistic models of inflation [33,34] with the potential profile similar to the Starobinsky inflation or with the residual cosmological constant suppressed as in the see-saw mechanism. This way is effective also in the description of quintessence [35]. The peculiarities of spontaneous breaking of global symmetry in the double measure theories were investigated in [36]. Some additional effects arise if one includes the specific interaction like $T^{\mu \nu} \nabla_{\mu} \nabla_{\nu} \phi$ as was studied in [37] devoted to a gravitational theory with a dynamical time, that results in extra conservation laws. As we recognize the two measure theories represent the different way of considering the shift of cosmological constant, surely.

We will consider cosmological consequences appearing because of various renormalization schemes of effective action for the inflaton as induced by one-loop corrections due to the bare non-minimal contact term, elsewhere.

\section{Acknowledgments}

This work is supported by Russian Foundation for Basic Research, grant \# 14-02-00096 (for VVK) and grant \# 15-02-03244 (for JaVB).

Note added. After the present paper has been written, authors of [38] have suggested a model of induced gravity with the scale invariance due to the non-minimal squared coupling of inflaton to the scalar curvature in the Jordan frame. The model treats the inflaton of Einstein frame as it possesses the global shift symmetry broken by a correction to the effective potential losing the scale invariance. Despite of similarity of this approach to our model we see that the motivations as well as the reasons for the global symmetry breaking are quite different.

\section{References}

[1] S. Weinberg, The Cosmological Constant Problem, Rev.Mod.Phys. 61 (1989) 1-23.

[2] S. Weinberg, The Cosmological constant problems, astro-ph/0005265.

[3] Y. Zel'dovich, The Cosmological constant and the theory of elementary particles, Sov.Phys.Usp. 11 (1968) 381-393. 
[4] J. Goldstone, Field Theories with Superconductor Solutions, Nuovo Cim. 19 (1961) 154-164.

[5] J. Goldstone, A. Salam, and S. Weinberg, Broken Symmetries, Phys.Rev. 127 (1962) 965-970.

[6] A. Nicolis, R. Rattazzi, and E. Trincherini, The Galileon as a local modification of gravity, Phys.Rev. D79 (2009) 064036, [arXiv:0811.2197].

[7] C. Deffayet, G. Esposito-Farese, and A. Vikman, Covariant Galileon, Phys.Rev. D79 (2009) 084003, [arXiv:0901.1314].

[8] N. Chow and J. Khoury, Galileon Cosmology, Phys.Rev. D80 (2009) 024037. [arXiv:0905.1325].

[9] C. Germani and A. Kehagias, UV-Protected Inflation, Phys.Rev.Lett. 106 (2011) 161302, [arXiv: 1012.0853].

[10] T. Kobayashi, M. Yamaguchi, and J. Yokoyama, Generalized G-inflation: Inflation with the most general second-order field equations, Prog.Theor.Phys. 126 (2011) 511-529, [arXiv: 1105.5723].

[11] A. De Felice, T. Kobayashi, and S. Tsujikawa, Effective gravitational couplings for cosmological perturbations in the most general scalar-tensor theories with second-order field equations, Phys.Lett. B706 (2011) 123-133, [arXiv:1108.4242].

[12] K. Van Acoleyen and J. Van Doorsselaere, Galileons from Lovelock actions, Phys.Rev. D83 (2011) 084025, [arXiv: 1102.0487].

[13] A. H. Guth, The Inflationary Universe: A Possible Solution to the Horizon and Flatness Problems, Phys.Rev. D23 (1981) 347-356.

[14] A. D. Linde, A New Inflationary Universe Scenario: A Possible Solution of the Horizon, Flatness, Homogeneity, Isotropy and Primordial Monopole Problems, Phys.Lett. B108 (1982) 389-393.

[15] A. Albrecht and P. J. Steinhardt, Cosmology for Grand Unified Theories with Radiatively Induced Symmetry Breaking, Phys.Rev.Lett. 48 (1982) 1220-1223.

[16] A. D. Linde, Chaotic Inflation, Phys.Lett. B129 (1983) 177-181.

[17] R. Kallosh, A. Linde, and D. Roest, A universal attractor for inflation at strong coupling, Phys.Rev.Lett. 112 (2014) 011303, [arXiv:1310.3950].

[18] A. A. Starobinsky, A New Type of Isotropic Cosmological Models Without Singularity, Phys.Lett. B91 (1980) 99-102.

[19] V. F. Mukhanov and G. V. Chibisov, Quantum Fluctuation and Nonsingular Universe. (In Russian), JETP Lett. 33 (1981) 532-535.

[20] F. L. Bezrukov and M. Shaposhnikov, The Standard Model Higgs boson as the inflaton, Phys.Lett. B659 (2008) 703-706, [arXiv:0710.3755].

[21] G. F. Giudice and H. M. Lee, Starobinsky-like inflation from induced gravity, arXiv:1402.2129.

[22] Planck Collaboration Collaboration, P. Ade et al., Planck 2013 results. XVI. Cosmological parameters, arXiv:1303.5076.

[23] BICEP2 Collaboration Collaboration, P. Ade et al., BICEP2 I: Detection Of B-mode Polarization at Degree Angular Scales, arXiv:1403.3985.

[24] Planck Collaboration Collaboration, R. Adam et al., Planck intermediate results. XXX. The angular power spectrum of polarized dust emission at intermediate and high Galactic latitudes, arXiv:1409.5738.

[25] A. Sakharov, Violation of CP Invariance, c Asymmetry, and Baryon Asymmetry of the Universe, Pisma Zh.Eksp.Teor.Fiz. 5 (1967) 32-35. 
[26] E. I. Guendelman and D. Singleton, Scalar gauge fields, arXiv:1402.7334.

[27] M. Kawasaki, M. Yamaguchi, and T. Yanagida, Natural chaotic inflation in supergravity, Phys.Rev.Lett. 85 (2000) 3572-3575, [hep-ph/0004243].

[28] R. Kallosh and A. Linde, New models of chaotic inflation in supergravity, JCAP 1011 (2010) 011, [arXiv: 1008.3375].

[29] K. Freese, J. A. Frieman, and A. V. Olinto, Natural inflation with pseudo - Nambu-Goldstone bosons, Phys.Rev.Lett. 65 (1990) 3233-3236.

[30] E. Guendelman and A. Kaganovich, Gravitational theory without the cosmological constant problem, Phys.Rev. D55 (1997) 5970-5980, [gr-qc/9611046].

[31] E. Guendelman and A. Kaganovich, The Principle of nongravitating vacuum energy and some of its consequences, Phys.Rev. D53 (1996) 7020-7025.

[32] E. Guendelman and A. Kaganovich, Dynamical measure and field theory models free of the cosmological constant problem, Phys.Rev. D60 (1999) 065004, [gr-qc/9905029].

[33] E. Guendelman, Scale invariance, new inflation and decaying lambda terms, Mod.Phys.Lett. A14 (1999) 1043-1052, [gr-qc/9901017].

[34] E. Guendelman, Scale invariance and vacuum energy, Mod.Phys.Lett. A14 (1999) 1397, [hep-th/0106084].

[35] E. Guendelman and A. Kaganovich, SSB of scale symmetry, fermion families and quintessence without the long range force problem, Int.J.Mod.Phys. A17 (2002) 417, [hep-th/0110040].

[36] E. Guendelman, Scale symmetry spontaneously broken by asymptotic behavior, Class.Quant.Grav. 17 (2000) 361-372, [gr-qc/9906025].

[37] E. Guendelman, Gravitational Theory with a Dynamical Time, Int.J.Mod.Phys. A25 (2010) 4081-4099, [arXiv: 0911.0178].

[38] C. Csaki, N. Kaloper, J. Serra, and J. Terning, Inflation from Broken Scale Invariance, arXiv: 1406.5192. 